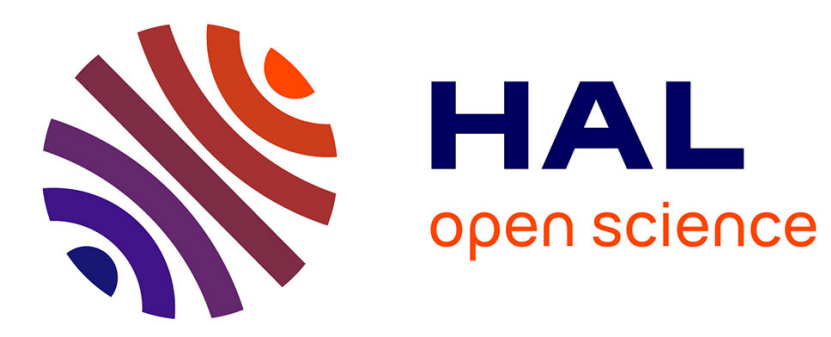

\title{
EEG analysis in anti-NMDA receptor encephalitis: Description of typical patterns
}

\author{
S. Jeannin-Mayer, N. André-Obadia, S. Rosenberg, C. Boutet, J. Honnorat, \\ J.C. Antoine, L. Mazzola
}

\section{- To cite this version:}

S. Jeannin-Mayer, N. André-Obadia, S. Rosenberg, C. Boutet, J. Honnorat, et al.. EEG analysis in anti-NMDA receptor encephalitis: Description of typical patterns. Clinical Neurophysiology, 2019, 130, pp.289 - 296. 10.1016/j.clinph.2018.10.017 . hal-03486411

\section{HAL Id: hal-03486411 \\ https://hal.science/hal-03486411}

Submitted on 20 Dec 2021

HAL is a multi-disciplinary open access archive for the deposit and dissemination of scientific research documents, whether they are published or not. The documents may come from teaching and research institutions in France or abroad, or from public or private research centers.
L'archive ouverte pluridisciplinaire HAL, est destinée au dépôt et à la diffusion de documents scientifiques de niveau recherche, publiés ou non, émanant des établissements d'enseignement et de recherche français ou étrangers, des laboratoires publics ou privés.

\section{(ㅇ)(1) $\$$}

Distributed under a Creative Commons Attribution - NonCommercial| 4.0 International 


\section{EEG analysis in anti-NMDA receptor encephalitis: description of typical patterns}

S. Jeannin-Mayer MD ${ }^{1}$, N. André-Obadia MD, $\mathrm{PhD}^{2}, \mathrm{~S}$. Rosenberg MD, $\mathrm{PhD}^{3}, \mathrm{C}$. Boutet $\mathrm{MD}, \mathrm{PhD}^{4}$, J. Honnorat MD, $\mathrm{PhD}^{5,6}$, JC. Antoine MD, $\mathrm{PhD}^{1}$, L. Mazzola MD, $\mathrm{PhD}^{1}$

${ }^{1}$ Department of Neurology, University Hospital Center of Saint-Etienne, France

${ }^{2}$ Department of Functional Neurology and Epileptology, Hospital for Neurology and

Neurosurgery Pierre Wertheimer, Hospices Civils de Lyon, Lyon, France.

${ }^{3}$ Department of Neurology and Clinical Neurophysiology, University Hospital of Clermont-

Ferrand, France

${ }^{4}$ Radiology department, University Hospital Center of Saint-Etienne, France

${ }^{5}$ Institut NeuroMyogène, Equipe Synaptopathies et Autoanticorps (SynatAc), INSERM U1217/UMR CRS 5310, University of Lyon, France

${ }^{6}$ French Reference Center on Paraneoplastic Neurological Syndrome, Hospices civils de Lyon, France

\section{Address correspondence to:}

Dr. Sophie Jeannin-Mayer

Department of Functional Neurology and Epileptology, University Hospital, Lyon, 59

Boulevard Pinel 69500 Bron

France

Tel.: +33427855192

Fax: +33472684945

E-mail: sophiejeannin@icloud.com

Dr. André-Obadia: e-mail: nathalie.obadia-andre@chu-lyon.fr

Dr. Rosenberg: e-mail: drosenberg@chu-clermontferrand.fr

Pr. Boutet: e-mail: claire.boutet@chu-st-etienne.fr

Pr. Honnorat: e-mail: jerome.honnorat@chu-lyon.fr

Pr. Antoine: e-mail: j.christophe.antoine@chu-st-etienne.fr

Dr. Mazzola: e-mail: lauremazzola@yahoo.fr 


\section{Abstract}

Objective. To describe different electroencephalogram (EEG) patterns and epileptic features in patients with anti- N-methyl-D-aspartate receptor encephalitis (anti-NMDARE), their timeline in the course of the disease, their correlation with clinical data and outcome.

Methods. We retrospectively analyzed EEG recordings between November 2007 and June 2016 in 24 consecutive patients.

Results. Three EEG patterns were described: Excessive Beta Activity range 14-20 Hz (EBA) in $71 \%$ of patients, Extreme Delta Brush (EDB) in $58 \%$ and Generalized Rhythmic Delta Activity (GRDA) in 50\%. They followed a chronological organization in the course of the disease: EBA appeared first, followed by EDB and then GRDA, as the median time of appearance for EBA, EDB and GRDA was respectively 10, 16.5 and 21.5 days. The presence of GRDA was strongly associated with concomitant abnormal movements ( $p<0.001$ ).

Conclusion. This study focuses on EEG and epileptic abnormalities in anti-NMDARE. Beyond EDB that were already reported (Schmitt et al., 2012), GRDA seems to be a very frequent pattern. Its rhythmic aspect should not be misinterpreted as seizure or status epilepticus, to avoid antiepileptic treatments intensification.

Significance. This study comforts the importance of EEG in anti-NMDARE, with a better description of EEG abnormalities for a better treatment management.

\section{Highlights}

- $\quad$ Three EEG patterns in anti NMDAR encephalitis, particularly Generalized Rhythmic Delta Activity (GRDA).

- GRDA were significantly associated with abnormal movements and differed from epileptic activity.

- Knowing EEG patterns in anti NMDAR encephalitis can help for diagnosis and drugs management.

Keywords: anti-NMDAR; encephalitis; EEG patterns; extreme delta brush; generalized rhythmic delta activity; excessive beta activity. 


\section{Introduction}

Anti-N-methyl D-aspartate receptor encephalitis (anti-NMDARE) is the most frequent human autoimmune encephalitis and is primarily directed against the NR1 subunit of the NMDAR (Dalmau et al., 2008). Clinical symptoms are initially marked by psychiatric manifestations, impaired cognition and seizures followed by movement disorders, loss of consciousness, and dysautonomia (Dalmau et al., 2011, 2008; Irani et al., 2010; Titulaer et al., 2013) The association of one of these symptoms and anti-NMDAR IgG antibodies detection made a definite diagnosis (Graus et al., 2016). As early treatment initiation improves the prognosis (Titulaer et al., 2013), early diagnosis is of important concern. Electroencephalogram (EEG) is non-invasive and easy to achieve, but data available concerning EEG features in antiNMDARE remains scarce. It was first used to characterize seizures, detect non-convulsive status epilepticus and help for the differential diagnosis of abnormal movements. An EEG pattern, named 'Extreme Delta Brush' (EDB) was described by Schmitt et al in 2012 (Schmitt et al., 2012) in 30\% of 23 patients with anti-NMDARE, and is one of the diagnosis criteria (Graus et al., 2016). Occasional case reports also described a generalized rhythmic delta activity in anti-NMDARE, but mostly without detailed description and with no common definition (Bayreuther et al., 2009; Gataullina et al., 2011; Johnson et al., 2010; Kadoya et al., 2015; Kirkpatrick et al., 2011). The aim of our study was to analyze retrospectively the different EEG patterns in 24 consecutive patients with anti-NMDARE, describe the timeline of EEG abnormalities, correlate them with the clinical and MRI data, treatments, and test whether any EEG pattern may reliably predict the outcome.

\section{Patients and methods}

This study was approved by the institutional review board of the University Claude Bernard Lyon 1 and Hospices Civils de Lyon.

\subsection{Patients}

Twenty-four patients with anti-NMDARE confirmed by CSF antibody detection in the reference center of Lyon (Viaccoz et al., 2014) and followed between November 2007 and June 2016 in the University Hospitals of Clermont-Ferrand, Lyon or Saint-Etienne were studied. All the patients had at least one EEG recording and one cerebral MRI. We reviewed age, gender, past medical history, date of disease onset $t=0$ (date of the first symptom described) and hospitalizations, MRI and EEG recordings. For each EEG recording, the presence of psychiatric symptoms, movement disorders, seizures, or dysautonomia was noticed. The modified Rankin Scale (mRS), Glasgow Scale (GS) scores, and treatments including immunotherapy, anti-epileptic and psychotropic drugs were recorded.

\subsection{EEG data}

Two hundred and ninety-four EEG recordings were performed including 234 EEG during at least 30 minutes and 60 cumulative days of prolonged EEG-monitoring. EEG monitoring $(>24 \mathrm{~h})$ were performed in 7 patients that presented coma state, continuous or prolonged 
abnormal movements and/or no clinical improvement despite two lines of immunotherapy. EEG studies were reviewed by two neurophysiologists (SJM and LM).

Electrodes were placed according to the 10/20 international system and at least 16 EEG channels were used. All EEG were in digital format with time-locked video. EEGs were evaluated for the presence or absence of i) excessive beta activity (EBA) (range 14-20 Hertz, 20-25 Hertz or higher than 25 Hertz); ii) slow waves ( $<8$ Hertz); iii) EDB, defined as delta activity 1-3 Hz, with superimposed burst of rhythmic beta frequency riding on each delta waves (1); iv) Generalized Rhythmic Delta Activity (GRDA) characterized by diffuse, synchronous and rhythmic delta activity; v) spikes, sharp waves, discharges or seizures. The video-EEG monitoring permitted to study the presence or absence of abnormal movements and their characteristics during each EEG pattern.

\section{$2.3 M R I$}

Brain MRIs ( $n=61$ ) were reviewed by 1 neuroradiologist $(C B)$ and 1 neurologist (SJM). We analyzed Diffusion-weighted imaging, T2-weighted FLAIR imaging, and T1-weighted imaging with contrast enhancement (gadolinium) sequences. The presence of abnormalities in the cerebral cortex, limbic structures, basal ganglia, cerebellum, and the presence of atrophy were analyzed.

\subsection{Statistical analysis}

Statistical analysis was performed with the SPSS 20TM software. A first analysis included all the performed EEG recordings (EEG analysis). The following variables were studied for each recording: presence of at least one EEG pattern, delay from disease onset, concomitant clinical features including age, gender, abnormal movements, seizures or dysautonomia, antiepileptic treatment and the mRS score. Multivariate logistic regression was used for the analysis with each EEG pattern as dependent variable and the other data as predictor independent variables. Independent variables were first analyzed by univariate logistic regression and then entered in the analysis if $p$ value was $<0.2$. The analysis was weighted for age and sex and to avoid bias due to the overrepresentation of patients with a large number of recordings, by the number of EEG recorded in each patient. In another analysis, the delay of appearance and disappearance, and the duration of each EEG abnormality and of clinical symptoms were measured and compared by the ANOVA test.

A second analysis (patient analysis), where electrophysiological data recorded for each patient within the first 2 months of follow-up were pooled without date distinction, was also performed for MRI abnormalities. Disease severity and outcome were appreciated by a modified Rankin Scale score $<3$ or $>3$ at 6,12 and 18 months of follow-up. 


\section{Results}

\subsection{Patient characteristics}

Among the 24 patients, 7 were children ( $<18$ years old). The median age was 20.7 years (range 1.5 to 62 ) and 21 of 24 patients (87\%) were female. Table 1 shows the detailed clinical features, diagnostic tests, mRS, treatments and outcome of these patients. A tumor was found in $29 \%(n=7)$, including ovarian teratoma $(n=5)$, small cell lung cancer $(n=1)$ and nonHodgkin lymphoma (NHL) $(n=1)$. Three patients died of infection, cancer or sudden death respectively.

Behavioral disorders were present at disease onset in all the patients leading to admission in psychiatric unit in $46 \%(\mathrm{n}=11)$ of cases. Neurologic symptoms included speech impairment, seizures, akinetic state and abnormal movements (mostly oro-facial dyskinesia). Seizures presented as focal seizures mostly with ictal consciousness alteration, orofacial or manual automatisms or motor symptoms (head deviation, facial or limb clonic seizures). Vigilance deterioration (GS score <12) led to the intensive care unit in $83 \%(n=20)$ of patients, with endotracheal intubation in $42 \%(n=10)$. In $24 \%(n=13)$ of patients a GS score $<8$ indicated coma. CSF was abnormal in $92 \%(n=22)$ of patients. The median number of brain MRI per patient was 2 (range 1-7). Antiepileptic drugs were administrated in all the patients during the course of their disease, however benzodiazepine could be given for epileptic or behavioral causes. 96\% $(n=23)$ of patients received psychoactive drugs and the same number immunotherapy.

\subsection{EEG abnormalities}

The median number of EEG recorded per patient was 8 (range 1-70) (Figure A.1).

\subsubsection{Epileptic abnormalities}

Seventy-five percent $(n=18)$ of patients had seizures. This number included $46 \%$ of patients $(n=11)$ with seizures during EEG recording and $29 \%$ of patients $(n=7)$ with seizures not recorded at the time of EEG but reported by medical observation. Most of seizures originated from the temporal lobe $(n=8)$, mainly characterized by consciousness alteration and orofacial or manual automatisms; or from fronto-central regions $(n=2)$, with predominant motor features such as tonic or clonic seizures. For one patient, seizures were multifocal. In $21 \%$ of patients $(n=5)$, electrographic seizures were observed, mainly in intubated or comatose patients $(n=3)$. No status epilepticus was recorded. Seizures appeared at a median delay of 9.5 days.

We recorded spikes in $62 \%(\mathrm{n}=15)$ of patients, in the temporal $(53 \% ; \mathrm{n}=8)$, frontal $(4 \% ; \mathrm{n}=1)$ or occipital $(4 \% ; n=1)$ regions. They were multifocal in 5 patients $(21 \%)$. One third $(n=7)$ of the patients in whom epileptic seizures were clinically reported had spikes on EEG recordings. 
Slow waves were recorded in all patients.

\subsubsection{Non-epileptic abnormalities}

Three patterns were identified, illustrated in Figure 1 (also Table 2).

-Excessive Beta Activity (EBA)

EBA 14-20 Hertz was observed in 71\% $(\mathrm{n}=17)$ of patients. It was diffuse or predominated in frontal areas. EBA range $>20$ Hertz was observed in all patients and differed by lower amplitude.

-Extreme Delta Brush (EDB)

EDB was observed in 58\% $(n=14)$ of patients and recorded in $6 / 7$ patients with EEG monitoring. It was usually diffuse but more pronounced frontally, sometimes transient or lasting several minutes. EDB was not modified by auditory and nociceptive stimuli.

\section{-Generalized Rhythmic Delta Activity (GRDA)}

GRDA was recorded in 50\% $(n=12)$ of patients including the $7 / 7$ patients with EEG monitoring. It was characterized by diffuse, synchronous, continuous and rhythmic delta slow waves or delta slow waves complexes. This activity was monotone, without acceleration or recruiting rhythm. It differed from seizures recorded in a same patient. GRDA were not modified by auditory or sensitive stimuli, persisted after antiepileptic drugs administration but were transiently altered by Propofol. It could occur continuously on 30-mins recordings but not on continuous EEG-monitoring, lasting from few minutes to several hours, at times replaced by periods of nonspecific irregular theta or delta activity, which lasted for hours, with intermittently superimposed faster $(>20 \mathrm{~Hz})$ activity, possibly secondary to sedative medication.

\subsection{Timeline of EEG abnormalities (Table 3)}

The timing of appearance, duration, and disappearance of EBA 14-20Hz, EDB and GDA had a non-random distribution (ANOVA test, $\mathrm{p}<0.001$ ). EBA appears first, followed by EDB and then GRDA with a respective median time of appearance of 10, 16.5 and 21.5 days. (Figure 2) EDB was more transient (median duration: 7 days) than others EEG patterns (EBA median duration: 17 days), whereas GRDA lasted longer (median duration: 29 days).

\subsection{Association of EEG abnormalities with anti-epileptic treatments and clinical symptoms}

\subsubsection{EBA}


Multivariate logistic regression in EEG analysis showed that EBA were significantly associated with EDB (OR $3.595 \%$ CI 1.6-7.7, p=0.002) and with barbiturate treatment (OR 5.1; 95\% CI 1.7-14.4; $\mathrm{p}=0.04$ ) and benzodiazepine (OR 3.1; 95\% CI 1.3-7.5, $\mathrm{p}=0.014$ ), and number of EEG per patient (OR 1.040 IC 95\% 1.017-1.053 p=0.001). No significant association was found with GDA, mRS score, or clinical symptoms ( $\mathrm{p}>0.99)$.

\subsubsection{EDB}

EDB were significantly associated with EBA (OR 3.6 95\% CI 1.7-7.8, p=0.002). No significant association was found with $\mathrm{mRS}$ score ( $\mathrm{p}>0.99$ ), treatment (Benzodiazepines OR 2.9 95\% CI 0.8-10.8 P=0.11; Barbiturates OR 2.9 95\% CI 0.9-9.2 p=0.072; Propofol OR 1.5 CI 95\% 0.6-3.7 $\mathrm{p}=0.4)$ and clinical symptoms $(\mathrm{p}>0.99)$. There was no significant correlation between EDB and the number of EEG recorded in each patient (OR 1.015 IC 95\% 0.994$1.037 \mathrm{p}=0.151$ )

\subsubsection{GRDA}

The presence of GRDA was strongly associated with concomitant abnormal movements (OR 4.7, 95\% CI: 2.3-9.3, p<0.001). GRDA was also associated with higher mRS score (OR 8.1, 95\% CI: 1.1-60.1, $\mathrm{p}=0.04)$. Interestingly, simultaneous recording of EMG activity showed that abnormal movements occurred at the same frequency and synchronously with GRDA activity (see Figure A.2 and Figure A.3). GRDA was associated with concomitant benzodiazepine administration (OR 2.3, 95\% CI: 1.0- 5.3, $\mathrm{p}=0.043$ ). There was no significant correlation between GRDA and the number of EEG per patient (OR 1.016 IC 95\% 0.999$1.034 \mathrm{p}=0.062$ )

\subsubsection{Seizures}

None of the three EEG patterns was correlated with seizures.

\subsection{EEG patterns and outcome}

Patient analysis found no significant correlation between recording of EBA, EDB, GRDA in the first 2 months of hospitalization and a mRS score $<3$ at 6,12 , and 18 months of follow-up (Chi-2 Test $\mathrm{p}>0.99$ ).

\subsection{EEG patterns and MRI findings}

Table A.1 summarizes MRI findings. Abnormalities were observed in 33\% ( $\mathrm{n}=8)$ of patients. Owing to their low number they could not be correlated with any of the EEG patterns.

\section{Discussion}

Our study is one of the largest to focus on EEG (including EEG monitoring) in patients with anti-NMDARE. We describe three EEG patterns: Excessive Beta Activity range 14-20 Hertz 
(EBA), Extreme Delta Brush (EDB) and Generalized Rhythmic Delta Activity (GRDA). A clear chronological organization in the course of the disease was evidenced, EBA appearing first, followed by EDB and then GRDA. None of these patterns was associated with seizures, but GRDA was associated with abnormal movements.

The peculiar aspect of EBA as we describe it, in range 14-20 Hertz, may be debatable because of the difficulty to distinguish it from excessive beta activity induced by treatments. Indeed, the presence of EBA was associated with concomitant benzodiazepine and barbiturate treatments. However, it is unlikely to be induced by these drugs as 4 patients demonstrated this pattern on the initial EEG before receiving benzodiazepines or barbiturates. In a same patient EBA 14-20Hertz differed in amplitude and frequency range from excessive beta activity induced by treatment that is described of smaller amplitude and faster range (until 25Hertz) (Schomer and Silva, 2012). Furthermore, the EBA ranging 14-20Hz observed in our patients had a similar appearance than the Beta rhythms superimposing the delta wave on EDB, as if EBA mingled with slow waves to form EDB; these two patterns are significantly associated in time. In their study Yildrim et al (Yildirim et al., 2018), also observed excessive beta activity in the first month of the disease. The generators of this pattern are probably cortical, as observed in cortical dysplasia (Raymond and Fish, 1996).

EDB is very peculiar to anti-NMDARE and reported in $16 \%$ to $41 \%$ of patients (Schmitt et al., 2012; Veciana et al., 2015; Yildirim et al., 2018; Zhang et al., 2017). We observed EDB in $58 \%$ of our patients, however, studied populations may be different and as it is a transient pattern (median duration 7 days), its recording may only depend on the length and frequency of EEG recordings. In our study, EDB were not associated with epileptic seizures or status epilepticus, as reported by Veciani et al (Veciana et al., 2015) and were not correlated with bad outcome. In literature, the prognosis of patients presenting EDB is still controversial as some of previous studies have shown that patients with EDB had worse outcome (da SilvaJúnior et al., 2014; Schmitt et al., 2012; VanHaerents et al., 2014) and others suggested favorable outcomes (Wang et al., 2015; Zhang et al., 2017). EDB are physiologically observed in premature neonatal EEG (Whitehead et al., 2017) and were also recently reported in FIRES (Febrile infection-related epilepsy syndrome) (Farias-Moeller et al., 2017). Their origin is unknown but several arguments suggest that they could be generated in the cortex. In neonates, delta brushes are described as an endogenous cortical pattern triggered by sensory input and seem to play an important role in the cerebral cortex development (Khazipov and Luhmann, 2006; Milh et al., 2007).

GRDA was found in 50\% of our patients, and was easier to record and identify because of its aspect easy to catch on EEG and its longer duration (median duration 29 days). In the literature this pattern is described in a wide range of etiologies in comatose patients (Rodriguez Ruiz et al., 2017; Trinka and Leitinger, 2015). In anti-NMDARE some studies have mentioned delta rhythmic activity or GRDA with no common definition, and sometimes poor EEG description (Schmitt et al., 2012; Yildirim et al., 2018; Zhang et al., 2017). Some reports seem to correspond to our description, showing repetition of a waveform with uniform morphology and duration, without an interval between consecutive waveforms, without 
variation of frequency or location, and prolonged from several minutes to several hours (da Silva-Júnior et al., 2014; Johnson et al., 2010; Kirkpatrick et al., 2011). But others, showed generalized rhythmic delta activity, with evolution in morphology, frequency (Kirkpatrick et al., 2011) or polymorphic or intermittent delta rhythms (Yildirim et al., 2018; Zhang et al., 2017). Some authors considered this activity as status epilepticus ((Gataullina et al., 2011; Johnson et al., 2010)) leading to increase antiepileptic drugs administration with their resulting adverse effects. Conversely, other reports considered rhythmic delta activities as non-epileptic since they were not improved by anti-epileptic treatments (Chanson et al., 2016; Gataullina et al., 2011; Kadoya et al., 2015; Probasco et al., 2014). In our study antiepileptic drugs could not stop GRDA. It could be depressed by propofol or barbiturates but reappeared at the same intensity several minutes after, despite the anesthetics infusion, and could disappear in some patients after several days, without any modifications of anti-epileptic drugs. Moreover, studies on critically ill or comatose patients found no association between GRDA and seizures, and the absence of clear evolution in frequency, location, or morphology of GRDA does not support an epileptic origin (Rodriguez Ruiz et al., 2017; Trinka and Leitinger, 2015). The significant association of GRDA with benzodiazepine treatment in our study probably reflects a misinterpretation of GRDA as seizures or status epilepticus by the clinicians in charge of the patients. So, it seems important to identify this pattern as non-ictal to avoid antiepileptic treatments intensification with their adverse effects especially as seizures mostly occurred at the beginning of the disease in our study (median time of appearance: 9.5 days), before GRDA apparition.

Abnormal movements were significantly correlated with the presence of GRDA. In several patients $(n=4)$, we observed simultaneous apparition of abnormal movements (dyskinesia) on video, and of GRDA on EEG (Figure 1 F). In some cases, the abnormal movement was synchronous with the frequency of GRDA (Figure A.3). The fact that GRDA did not disappear after curare infusion, in contrast with the abnormal movement, proves that they are not the result of artifacts generated by rhythmic movements (Figure A.2). Their strong association with abnormal movements suggests that they may be generated in the basal ganglia. Imaging has demonstrated the implication of basal ganglia in anti-NMDARE (Kataoka et al, 2009; Maeder-Ingvar et al, 2011). Indeed, thalamic, caudate nucleus and lenticular nucleus abnormal signals on brain MRI, as in our study, are frequent in this disorder (Dalmau et al., 2011; Heine et al., 2015) and striatal hypermetabolism has also been reported on FDG PET scanner (Baumgartner et al., 2013; Maeder-Ingvar et al, 2011). GRDA are described in several other clinical situations mainly with basal ganglia dysfunctions such as midline brain lesions, subcortical lesions, Creutzfeldt-Jakob disease, diffuse Lewy body disease (Rodriguez et al, 2016) or in superficial stage of coma in patients with deep midline lesions affecting thalamo-cortical projection or in anoxia (Trinka et al, 2016).

Another interesting finding of our study is the temporal progression of each pattern and its correlation with the disease progression. Irani et al (Irani et al., 2010), has hypothesized that the disease progressed anatomically from the cortical to subcortical structures (in particular the basal ganglia). They reported a mean time lag of 10 to 20 days between the disease onset marked by cognitive and psychiatric symptoms, seizures and cortical MRI lesions, and the 
later appearance of features indicating basal ganglia and brainstem involvement such as movement disorders, loss of consciousness, dysautonomia and subcortical MRI lesions. In the same way EBA and EDB, which are probably generated in cortical areas, occur before 20 days of disease evolution while GRDA, which appears after 20 days, are correlated with abnormal movements, may be related to basal ganglia dysfunction. Although time course of clinical symptoms can vary from one patient to another, and the absence of MRI or neuropathological well-established data showing this progression,

Seventy-five percent of patients had seizures, predominantly at a relatively early stage of the disease (median delay of 9.5 days). In $21 \%$ of patients, electrographic seizures were observed without any clinical manifestation, strengthening the need of prolonged EEG monitoring to adapt antiepileptic drugs administration.

To conclude, EDB and GRDA are two frequent and prominent EEG patterns of antiNMDARE. Although not specific, these patterns are suggestive and their recognition can help to an earlier diagnosis of the disorder. Frequent EEG monitoring, from the onset of disease, is a key step to detect these patterns and electrographic seizures. Moreover, GRDA must not be misinterpreted as seizures or status epilepticus, leading to increase antiepileptic drugs administration and so worsening awareness. Finally, temporal organization and timeline of EEG patterns bring additional electrophysiological support to the hypothesis of a temporal progression of the disease from an initial predominantly cortical impairment to subcortical structure and especially basal ganglia dysfunction (Figure 3).

\section{Limits}

One limit of our retrospective study was the absence of long EEG monitoring for each patient that probably underestimates the presence of electrographic abnormalities. Similarly, the median number of cerebral MRI per patients was only 2. For a better understanding of the disease, especially to support the pathological progression hypothesis, it will be interesting to combine EEG monitoring to MRI monitoring with N-acétyl aspartate level analyzing in spectroscopy (Maeder-Ingvar et al, 2011) and even more FDG PET scanner when achievable.

\section{Supplementary Material}

Figure A.1, Figure A.2, Figure A.3.

Table A.1. 


\section{Declaration of interest}

Dr Jeannin-Mayer had full access to all the data in the study and takes responsibility for the integrity of the data and the accuracy of the data analysis.

The statistical analysis was performed by Professor Antoine

Dr. Jeannin-Mayer reports no disclosures.

Dr André-Obadia reports no disclosures.

Dr. Rosenberg received in 2017 honoraria from serving on the scientific advisory board of Novartis Pharma.

Pr. Boutet reports no disclosures.

Pr. Honnorat served on the scientific advisory board for BMS, received research support from CSL Behring and receives revenue from Athena Diagnostics, Euroimmun and RAVO Diagnostika for a patent.

Pr. Antoine received funding for travels from LFB and CSL Behring and honoraria for scientific consulting from Pfeizer and from a license on diagnostic test for the detection of anti-CRMP5 antibodies.

Dr. Mazzola reports no disclosures 


\section{References}

Baumgartner, A., Rauer, S., Mader, I., Meyer, P.T., 2013. Cerebral FDG-PET and MRI findings in autoimmune limbic encephalitis: correlation with autoantibody types. J. Neurol. 260, 2744-2753.

Bayreuther, C., Bourg, V., Dellamonica, J., Borg, M., Bernardin, G., Thomas, P., 2009. Complex partial status epilepticus revealing anti-NMDA receptor encephalitis. Epileptic. Disord. 11(3), 261-265.

Chanson, E., Bicilli, É., Lauxerois, M., Kauffmann, S., Chabanne, R., Ducray, F., et al., 2016. Anti-NMDA-R encephalitis: Should we consider extreme delta brush as electrical status epilepticus? Neurophysiol. Clin. Neurophysiol. 46, 17-25.

da Silva-Júnior, F.P., Castro, L.H.M., Andrade, J.Q., Bastos, C.G., Moreira, C.H., Valério, R.M.F., et al., 2014. Serial and prolonged EEG monitoring in anti-N-Methyl-dAspartate receptor encephalitis. Clin. Neurophysiol. 125, 1541-1544.

Dalmau, J., Gleichman, A.J., Hughes, E.G., Rossi, J.E., Peng, X., Lai, M., et al, 2008. AntiNMDA-receptor encephalitis: case series and analysis of the effects of antibodies. Lancet Neurol. 7, 1091-1098.

Dalmau, J., Lancaster, E., Martinez-Hernandez, E., Rosenfeld, M.R., Balice-Gordon, R., 2011. Clinical experience and laboratory investigations in patients with anti-NMDAR encephalitis. Lancet Neurol. 10, 63-74.

Farias-Moeller, R., Bartolini, L., Staso, K., Schreiber, J.M., Carpenter, J.L., 2017. Early ictal and interictal patterns in FIRES: The sparks before the blaze. Epilepsia 58, 13401348.

Gataullina, S., Plouin, P., Vincent, A., Scalais, E., Nuttin, C., Dulac, O., 2011. Paroxysmal EEG pattern in a child with N-methyl-d-aspartate receptor antibody encephalitis: Case Report. Dev. Med. Child Neurol. 53, 764-767.

Graus, F., Titulaer, M.J., Balu, R., Benseler, S., Bien, C.G., Cellucci, T., et al., 2016. A clinical approach to diagnosis of autoimmune encephalitis. Lancet Neurol. 15, 391404.

Heine, J., Prüss, H., Bartsch, T., Ploner, C.J., Paul, F., Finke, C., 2015. Imaging of autoimmune encephalitis - Relevance for clinical practice and hippocampal function. Neuroscience 309, 68-83.

Irani, S.R., Bera, K., Waters, P., Zuliani, L., Maxwell, S., Zandi, M.S., et al., 2010. N-methyld-aspartate antibody encephalitis: temporal progression of clinical and paraclinical observations in a predominantly non-paraneoplastic disorder of both sexes. Brain 133, 1655-1667.

Johnson, N., Henry, C., Fessler, A.J., Dalmau, J., 2010. Anti-NMDA receptor encephalitis causing prolonged nonconvulsive status epilepticus. Neurology 75, 1480-1482.

Kadoya, M., Onoue, H., Kadoya, A., Ikewaki, K., Kaida, K., 2015. Refractory Status Epilepticus Caused by Anti-NMDA Receptor Encephalitis that Markedly Improved Following Combination Therapy with Rituximab and Cyclophosphamide. Intern. Med. 54, 209-213.

Kataoka H, Dalmau J, Taoka T, Ueno S. Reduced N-acetylaspartate in the basal ganglia of a patient with anti-NMDA receptor encephalitis. Mov Disord 2009; 24:784-6.

Khazipov, R., Luhmann, H.J., 2006. Early patterns of electrical activity in the developing cerebral cortex of humans and rodents. Trends Neurosci. 29, 414-418.

Kirkpatrick, M.P., Clarke, C.D., Sonmezturk, H.H., Abou-Khalil, B., 2011. Rhythmic delta activity represents a form of nonconvulsive status epilepticus in anti-NMDA receptor antibody encephalitis. Epilepsy Behav. 20, 392-394.

Maeder-Ingvar M, Prior JO, Irani SR, Rey V, Vincent A, Rossetti AO. FDG-PET 
hyperactivity in basal ganglia correlating with clinical course in anti-NDMA-R antibodies encephalitis. J Neurol Neurosurg Psychiatry 2011; 82:235-236.

Milh, M., Kaminska, A., Huon, C., Lapillonne, A., Ben-Ari, Y., Khazipov, R., 2007. Rapid Cortical Oscillations and Early Motor Activity in Premature Human Neonate. Cereb. Cortex 17, 1582-1594.

Probasco, J.C., Benavides, D.R., Ciarallo, A., Sanin, B.W., Wabulya, A., Bergey, G.K., et al., 2014. Electroencephalographic and fluorodeoxyglucose-positron emission tomography correlates in anti-N-methyl-d-aspartate receptor autoimmune encephalitis. Epilepsy Behav. Case Rep. 2, 174-178.

Raymond, A.A., Fish, D.R., 1996. EEG Features of Focal Malformations of Cortical Development: J. Clin. Neurophysiol. 13, 495-506.

Rodriguez Ruiz, A., Vlachy, J., Lee, J.W., Gilmore, E.J., Ayer, T., Haider, H.A., et al., 2017. Association of Periodic and Rhythmic Electroencephalographic Patterns With Seizures in Critically Ill Patients. JAMA Neurol. 74, 181.

Schmitt, S.E., Pargeon, K., Frechette, E.S., Hirsch, L.J., Dalmau, J., Friedman, D., 2012. Extreme delta brush: A unique EEG pattern in adults with anti-NMDA receptor encephalitis. Neurology 79, 1094-1100.

Schomer, D.L., Silva, F.L. da, 2012. Niedermeyer's Electroencephalography: Basic Principles, Clinical Applications, and Related Fields. Lippincott Williams \& Wilkins.

Titulaer, M.J., McCracken, L., Gabilondo, I., Armangué, T., Glaser, C., Iizuka, T., et al., 2013. Treatment and prognostic factors for long-term outcome in patients with antiNMDA receptor encephalitis: an observational cohort study. Lancet Neurol. 12, 157165.

Trinka, E., Leitinger, M., 2015. Which EEG patterns in coma are nonconvulsive status epilepticus? Epilepsy Behav. 49, 203-222.

VanHaerents, S., Stillman, A., Inoa, V., Searls, D.E., Herman, S.T., 2014. Early and persistent 'extreme delta brush' in a patient with anti-NMDA receptor encephalitis. Epilepsy Behav. Case Rep. 2, 67-70.

Veciana, M., Becerra, J.L., Fossas, P., Muriana, D., Sansa, G., Santamarina, E., et al., 2015. EEG extreme delta brush: An ictal pattern in patients with anti-NMDA receptor encephalitis. Epilepsy Behav. 49, 280-285.

Viaccoz, A., Desestret, V., Ducray, F., Picard, G., Cavillon, G., Rogemond, V., et al., 2014. Clinical specificities of adult male patients with NMDA receptor antibodies encephalitis. Neurology 82, 556-563.

Wang, J., Wang, K., Wu, D., Liang, H., Zheng, X., Luo, B., 2015. Extreme delta brush guides to the diagnosis of anti-NMDAR encephalitis. J. Neurol. Sci. 353, 81-83.

Whitehead, K., Pressler, R., Fabrizi, L., 2017. Characteristics and clinical significance of delta brushes in the EEG of premature infants. Clin. Neurophysiol. Pract. 2, 12-18.

Yildirim, M., Konuskan, B., Yalnizoglu, D., Topaloglu, H., Erol, I., Anlar, B., 2018. Electroencephalographic findings in anti- $\mathrm{N}$-methyl- $\mathrm{d}$-aspartate receptor encephalitis in children: A series of 12 patients. Epilepsy Behav. 78, 118-123.

Zhang, Y., Liu, G., Jiang, M.D., Li, L.P., Su, Y.Y., 2017. Analysis of electroencephalogram characteristics of anti-NMDA receptor encephalitis patients in China. Clin. Neurophysiol. 128, 1227-1233. 


\section{Figures legends}

Figure 1. Three EEG patterns were identified in anti NMDAR encephalitis.

High pass filter $0.530 \mathrm{~Hz}$; Low pass filter $50 \mathrm{~Hz}$.

(A;B) Excessive Beta activity: (A) Excessive beta activity in 16 Hertz frequencies, bilateral in frontal areas. (B) Excessive beta activity in 19 Hertz frequencies, diffuse and bilateral. This pattern was recorded in the absence of benzodiazepine treatment prior or during EEG recording; (C;D) Extreme Delta Brush: (C) EDB consisting of slow waves with superimposed beta activity at 11 Hertz frequencies, bilateral in frontal areas (D) EDB with slow waves and diffuse superimposed beta activity at $14 \mathrm{Hertz}$ (E;F) Generalized Rhythmic Delta Activity: (E) continuous slow wave in delta range, synchronous, diffuse, rhythmic without spatial or temporal recruitment (F) GRDA associated with muscular artifacts on the left side in the same periodicity corresponding to concomitant abnormal movements.

Figure 2. Temporal organization of EEG patterns.

Appearance timing for each EEG pattern (Excessive Beta Activity range 14-20 Hz, Extreme Delta Brush, Generalized Delta Activity) and clinical symptoms.

Figure 3. Timeline of EEG patterns and clinical symptoms.

Diagram detailing the temporal evolution of EEG abnormalities and symptoms observed in patients with anti-NMDAR encephalitis with physiopathological hypothesis. 


\section{Figure 1:}

A.

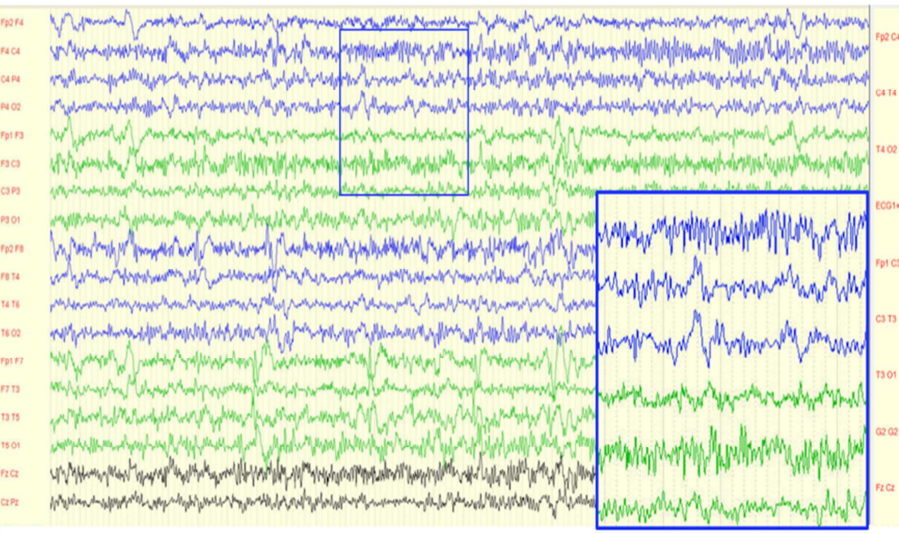

C.

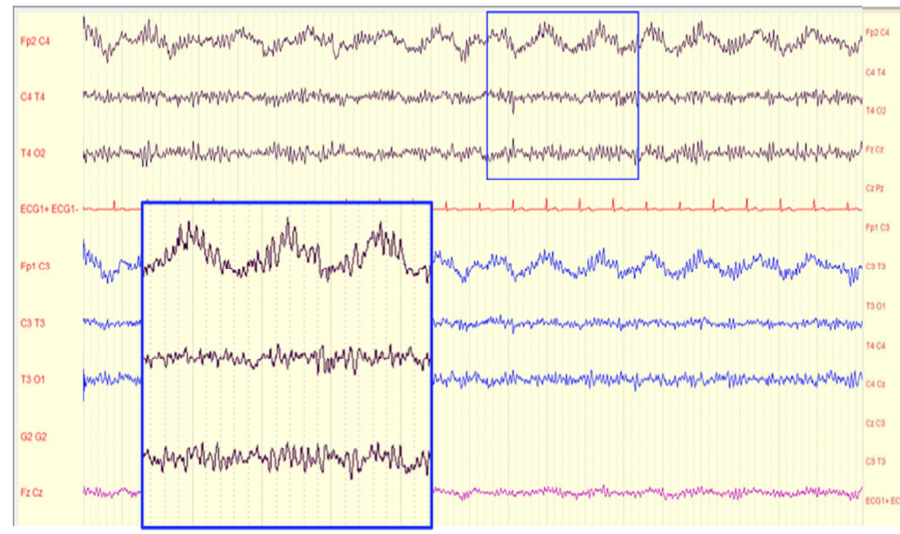

E.

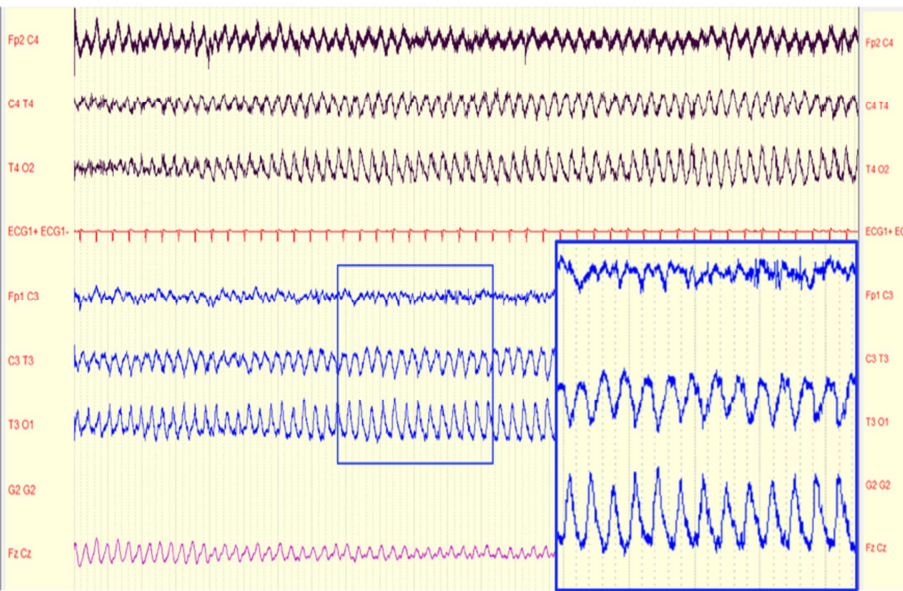

B.

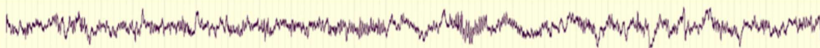

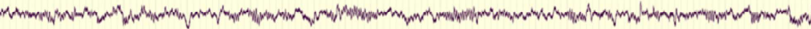

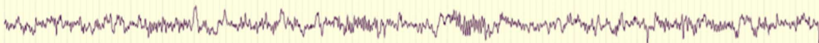

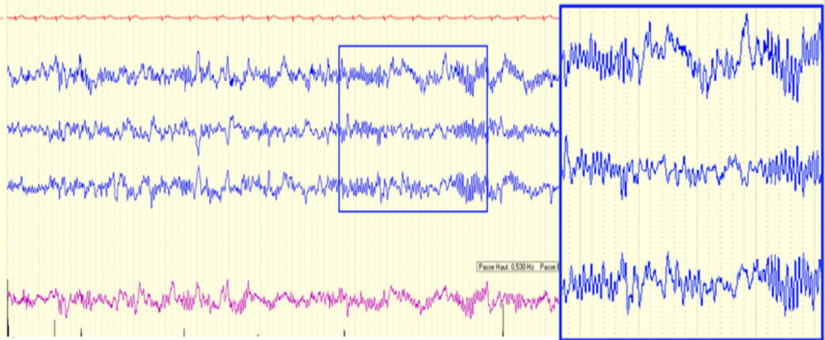

D.

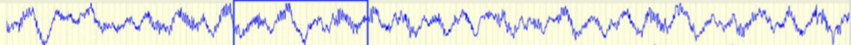

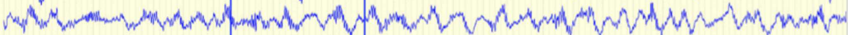

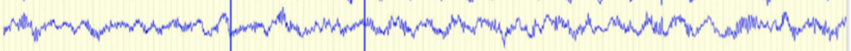

monment

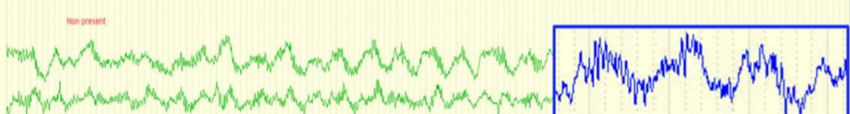

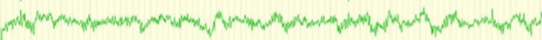

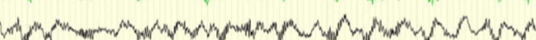

W.

(1)

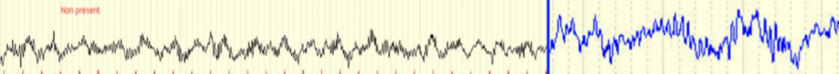

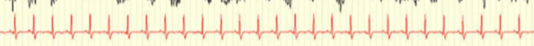

F.

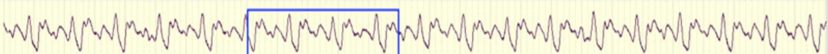

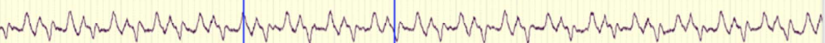

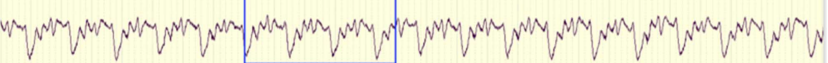

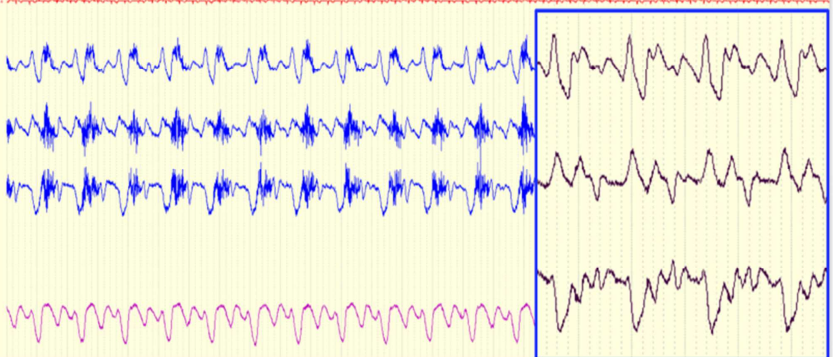


Figure 2.

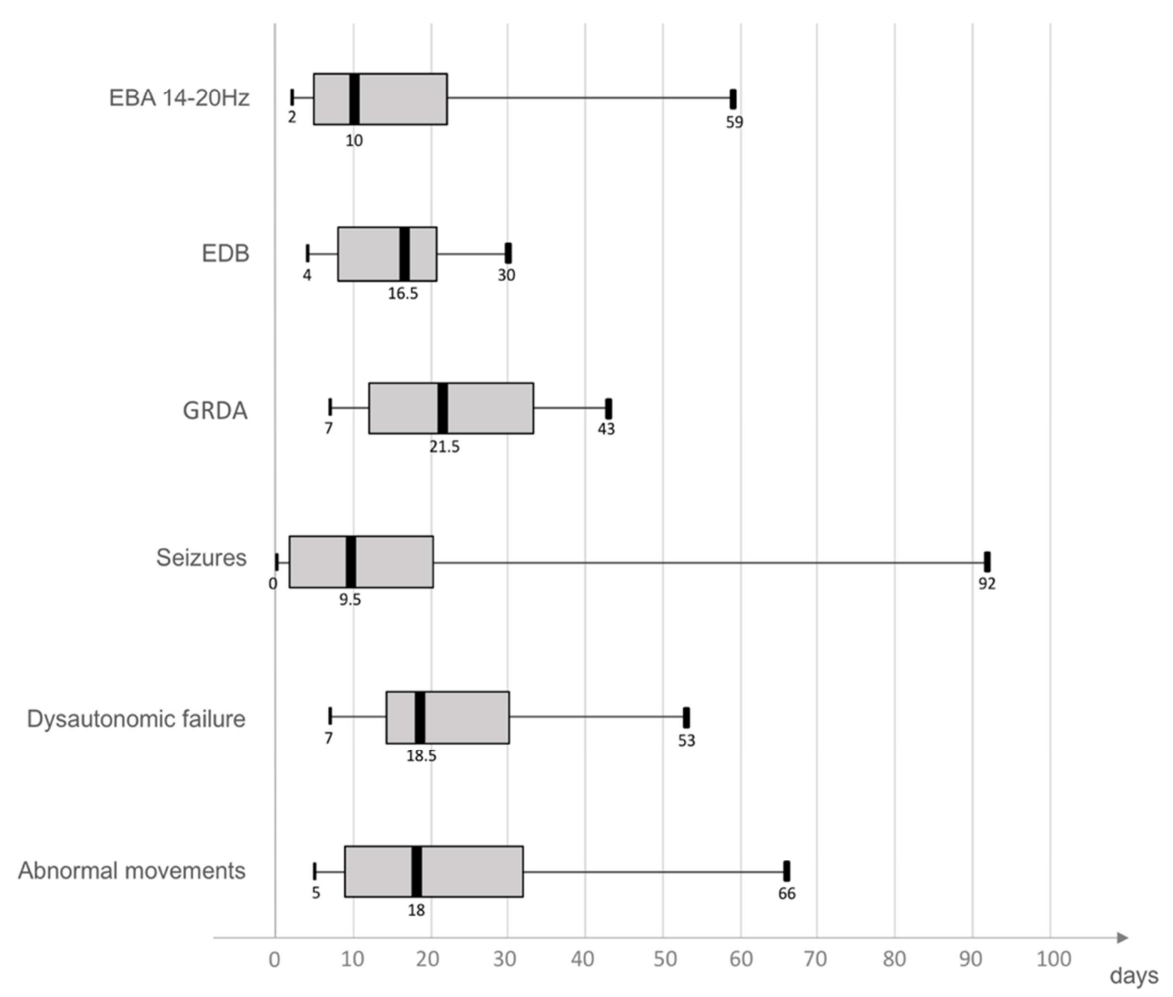




\section{Figure 3.}

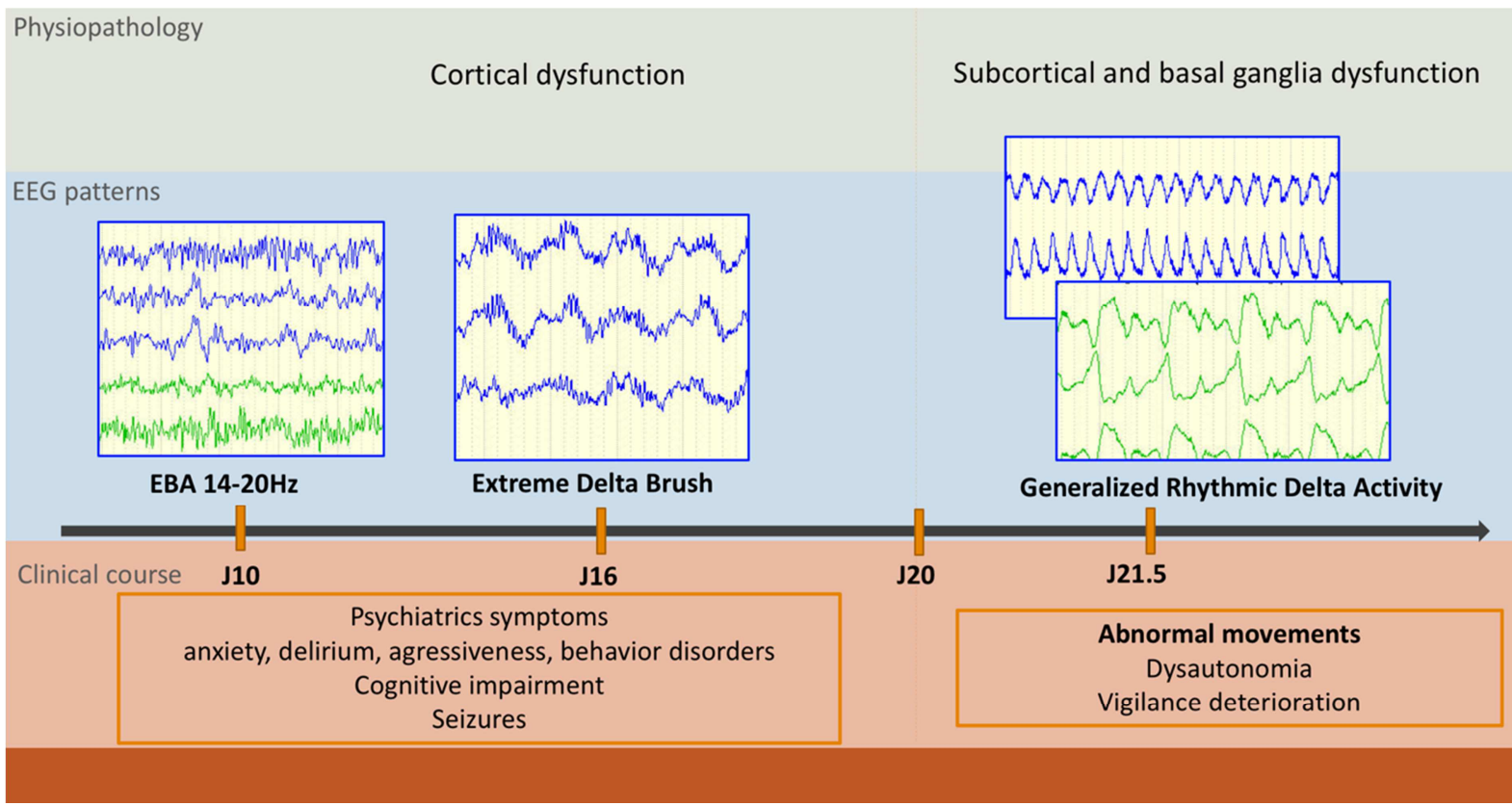


Table 1. Clinical features, diagnostic tests, treatments and outcome

\begin{tabular}{|c|c|}
\hline Clinical features & $\%(\mathrm{n}=24)$ \\
\hline Median Age & $20.7(1.5-62)$ \\
\hline Female & $87.5(21)$ \\
\hline Associated tumor & $29.2(7)$ \\
\hline - ovarian teratoma & $20.8(5)$ \\
\hline - small cell lung cancer & $4.2(1)$ \\
\hline - Non hodgkin lymphoma & $4.2(1)$ \\
\hline \multicolumn{2}{|l|}{ Hospitalization } \\
\hline - in psychiatry unit & $45.8(11)$ \\
\hline - in intensive care unit & $83.3(20)$ \\
\hline Hospitalization delay (median) & 133 days \\
\hline Behavior disorders & $100(24)$ \\
\hline - agitation / agressiveness & $100(24)$ \\
\hline - eating disorders & $45.8(11)$ \\
\hline Psychiatric symptoms (anxiety, & $95.8(23)$ \\
\hline \multirow{2}{*}{\multicolumn{2}{|c|}{$\begin{array}{l}\text { delusional thoughts, hallucinations) } \\
\text { Neurological symptoms }\end{array}$}} \\
\hline & \\
\hline - Abnormal movement & $87.5(21)$ \\
\hline Oro-facial & $79.1(19)$ \\
\hline Upper limb & $58.3(14)$ \\
\hline Lower limb & $45.8(11)$ \\
\hline Chest & $33.3(8)$ \\
\hline - Seizures & $75(18)$ \\
\hline - Language disorders / mutism & $91.6(22)$ \\
\hline Dysautonomic failure & $58.3(14)$ \\
\hline \multicolumn{2}{|l|}{ CSF } \\
\hline - pleiocytosis & $66.7(16)^{\circ}$ \\
\hline - elevated protein concentration & $25(6)^{\circ}$ \\
\hline - oligoclonal bands & $66.7(16)^{*}$ \\
\hline \multicolumn{2}{|l|}{ Treatments } \\
\hline Psychoactive drugs & $95.8(23)$ \\
\hline - anxiolytics & $91.6(22)$ \\
\hline - antipsychotics & $79.1(19)$ \\
\hline Antiepileptic drugs & $100(24)$ \\
\hline - benzodiazepin & $95.8(23)$ \\
\hline - barbiturates & $16.6(4)$ \\
\hline - levetiracetam & $66.7(16)$ \\
\hline - lacosamide & $29.2(7)$ \\
\hline - lamotrigin & $5.9(2)$ \\
\hline - perampanel & $16.6(4)$ \\
\hline Intubation & $41.7(10)$ \\
\hline Immunotherapy & $95.8(23)$ \\
\hline - Steroids & $66.7(16)$ \\
\hline - Immunoglobulin & $83.3(20)$ \\
\hline - Rituximab & $50(12)$ \\
\hline - Cyclophosphamide & $29.2(7)$ \\
\hline - Mycophenolate mofetil & $50(12)$ \\
\hline - Others & $16.6(4)$ \\
\hline \multicolumn{2}{|l|}{ Outcome } \\
\hline Death & $12.5(3)$ \\
\hline mRS score & median score (range) \\
\hline mRS score at 3 months of follow-up & $4(1-6)$ \\
\hline mRS score at 6 months of follow-up & $3(0-6)$ \\
\hline mRS score at 12 months of follow-up & $1(0-6)$ \\
\hline
\end{tabular}


$\underline{\text { Table 2. EEG findings }}$

\begin{tabular}{|c|c|}
\hline & $\%(n)$ \\
\hline $\begin{array}{l}\text { Number of EEG per patient (median) } \\
\text { Abnormal }\end{array}$ & $\begin{array}{c}8 \\
100(24) \\
\end{array}$ \\
\hline \multicolumn{2}{|l|}{ Non epileptic abnormalities } \\
\hline Slow waves & $100(24)$ \\
\hline Excessive beta activity & $100(24)$ \\
\hline - 14-20 Hertz & $70.8(17)$ \\
\hline - 20-25 Hertz & $100(24)$ \\
\hline$->25$ Hertz & $33.3(8)$ \\
\hline EDB & $58.3(14)$ \\
\hline GRDA & $50(12)$ \\
\hline \multicolumn{2}{|l|}{ Epileptic abnormalities } \\
\hline Spikes & $62.5(15)$ \\
\hline Electrographic seizures & $45.8(11)$ \\
\hline Status epilepticus & $0(0)$ \\
\hline Normal state II sleep figures & $54.2(13)$ \\
\hline
\end{tabular}

Abreviations: EDB Extreme Delta Brush

GDA Generalized Rhythmic Delta Activity 
$\underline{\text { Table 3. Appearance, Duration and Disappearance of patterns and clinical symptoms (in days) }}$

\begin{tabular}{|c|c|c|c|c|c|c|c|}
\hline & & \multicolumn{3}{|c|}{ Pattern EEG } & \multicolumn{3}{|c|}{ Clinical symptoms } \\
\hline & & EBA $14-20 \mathrm{~Hz}$ & EDB & GRDA & Seizures & Abnormal movements & Dysautonomia failure \\
\hline \multirow{5}{*}{ Appearance } & Median & 10 & 16.5 & 21.5 & 9.5 & 18 & 18.5 \\
\hline & Mean & 15 & 14.7 & 23.5 & 16.4 & 20.4 & 21.9 \\
\hline & SD & 14.3 & 8 & 12.6 & 22.4 & 15.3 & 13.2 \\
\hline & Min & 2 & 4 & 7 & 0 & 5 & 7 \\
\hline & Max & 59 & 30 & 43 & 92 & 66 & 53 \\
\hline \multirow{5}{*}{ Duration } & Median & 17 & 7 & 29 & 7.5 & 32 & 16 \\
\hline & Mean & 34.1 & 18.3 & 73.5 & 31.7 & 73.5 & 53 \\
\hline & SD & 49 & 31.2 & 119.7 & 83.4 & 119.1 & 101.5 \\
\hline & Min & 5 & 2 & 7 & 1 & 2 & 1 \\
\hline & Max & 195 & 115 & 411 & 357 & 497 & 385 \\
\hline \multirow{5}{*}{ Disappearance } & Median & 29 & 25.5 & 51 & 20 & 59 & 43 \\
\hline & Mean & 49.1 & 33 & 98.4 & 48.1 & 94.6 & 74.9 \\
\hline & SD & 47.4 & 29.5 & 127.3 & 91.5 & 115.9 & 101 \\
\hline & Min & 9 & 8 & 27 & 3 & 11 & 8 \\
\hline & Max & 198 & 124 & 454 & 400 & 505 & 399 \\
\hline
\end{tabular}

$\mathrm{SD}=$ standard deviation 